\title{
An Empirical Jet-Surface Interaction Noise Model with Temperature and Nozzle Aspect Ratio Effects
}

\author{
Cliff Brown * \\ NASA Glenn Research Center, Cleveland, OH, 44135, USA
}

\begin{abstract}
An empirical model for jet-surface interaction (JSI) noise produced by a round jet near a flat plate is described and the resulting model evaluated. The model covers unheated and hot jet conditions $\left(1 \leq T_{T, R} \leq 2.7\right)$ in the subsonic range $\left(0.5 \leq M_{a} \leq 0.9\right)$, surface lengths $0.6 \leq x_{T E} / D_{j} \leq 10$, and surface standoff distances $\left(0 \leq h_{T E} / x_{T E} \leq 1\right)$ using only second-order polynomials to provide predictable behavior. The JSI noise model is combined with an existing jet mixing noise model to produce exhaust noise predictions. Fit quality metrics and comparisons to between the predicted and experimental data indicate that the model is suitable for many system level studies. A first-order correction to the JSI source model that accounts for the effect of nozzle aspect ratio is also explored. This correction is based on changes to the potential core length and frequency scaling associated with rectangular nozzles up to 8:1 aspect ratio. However, more work is needed to refine these findings into a formal model.
\end{abstract}

\section{Nomenclature}

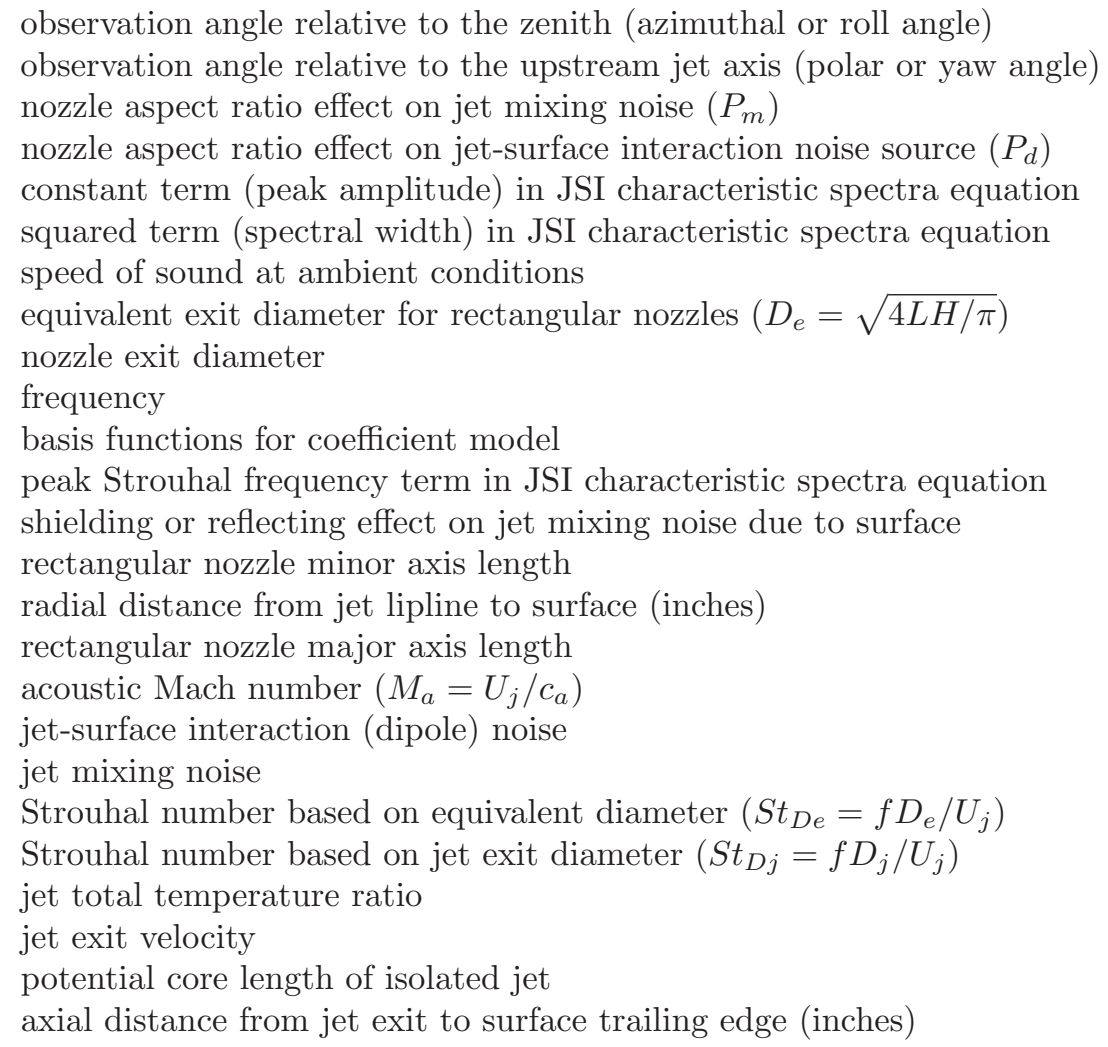

*Research Engineer, Acoustics Branch, 21000 Brookpark Rd., AIAA Member. 


\section{Introduction}

ThE demand for more efficient aircraft has led to proposed changes in the design of current and future fleet.

Many current commercial aircraft, for example, use high bypass ratio engines to extract more propulsive force from the same core with a lower exhaust velocity and, therefore, reduced engine exhaust noise. Future designs, such as the NASA TurboElectric Distributed Propulsion concept, integrate the engines into the airframe for better overall aerodynamic performance while maintaining an ultra high bypass ratio engine system . Proposed commercial supersonic aircraft also require tight engine/airframe integration to meet the performance requirements at cruise while minimizing the sonic boom in order to be viable. Therefore, it is necessary to understand the implications to performance, efficiency, and noise of engine exhaust near airframe surfaces.

Modern jet exhaust noise prediction tools provide a reasonable estimate when the considering an isolated round jet (e.g [?,[2],[3]). However, installation effects caused by nearby airframe surface have not generally been included in these predictions. This is, in part, because experimental data suitable for developing and validating general prediction tools has not been widely available. A recent series of experiments at the NASA Glenn Research Center supported by the NASA Fundamental Aeronautics Program Fixed Wing and High Speed Projects, have been conducted to provide a noise ${ }^{4-6}$ and flow ${ }^{7}$ database appropriate for developing and validating empirical and physics based jet-surface interaction (JSI) noise prediction methods. These experiments employ a common and simple geometry, a flat plate and a round convergent nozzle (Figure 1), to create general jet-surface configuration which is applicable in many situations. The jet exhaust conditions (Table 1) and surface position (Table 2) were varied parametrically to extract the individual effects of different variables in the three regions of interest: JSI as a noise source, surface shielding jet mixing noise, and the transition where both the JSI noise source and shielding effect are present. These data contain the information required to develop an empirical model for the JSI noise produced when a round nozzle exhausts near a surface.

An empirical model can quickly provide a first-order approximation to the overall effect of varying different input variables through a range defined by the underlying source data. Although lacking flexibility to extend too far from this range, empirical models are commonly used in system level studies where the combined effects of many small changes are considered in search for an optimum point and computational speed is valued. In 2014, an empirical model (JSI14) for the JSI noise source created by a round unheated jet near a flat surface was developed as the basis for a more comprehensive model that could eventually incorporate jet temperature, nozzle aspect ratio, and flight effects. ${ }^{8}$ This JSI source model is now simplified and extended to include hot jet exhaust conditions (JSI15).

The effect of nozzle aspect ratio on the JSI noise source has been measured in a related set of experiments ${ }^{9}$ . Far-field noise data were acquired using 2:1, 4:1, and 8:1 aspect ratio rectangular nozzles at the unheated exhaust conditions (setpoints 3,5, and 7 in Table 1) using a subset of the surface positions listed in Table 2. These data are interrogated to form a first-order empirical correction to the JSI15 model (round nozzle) that accounts for nozzle aspect ratio.

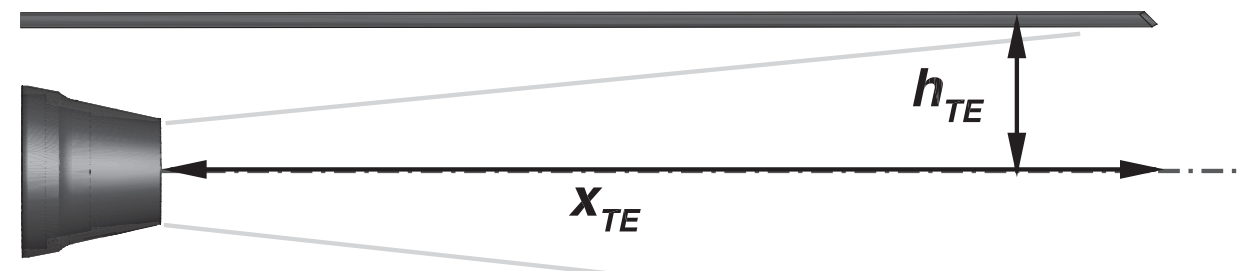

Figure 1. Schematic showing the configuration tested with the nomenclature used to describe the surface and observed locations. Note that $x_{T E}$ and $h_{T E}$ have dimensions and are nondimensionalized appropriate to the individual models. 


\begin{tabular}{|c|c|c|c|c|}
\hline Setpoint & Nozzle Pressure Ratio & $T_{T, R}$ & $M_{a}$ & Mass Flow $(\mathrm{kg} / \mathrm{s})$ \\
\hline 3 & 1.197 & 1.0 & 0.5 & 0.44 \\
\hline 5 & 1.436 & 1.0 & 0.7 & 0.65 \\
\hline 7 & 1.860 & 1.0 & 0.9 & 0.91 \\
\hline 23 & 1.103 & 1.81 & 0.5 & 0.24 \\
\hline 32 & 1.079 & 2.43 & 0.5 & 0.19 \\
\hline 27 & 1.360 & 1.91 & 0.9 & 0.43 \\
\hline 36 & 1.273 & 2.43 & 0.9 & 0.33 \\
\hline 46 & 1.227 & 2.75 & 0.9 & 0.28 \\
\hline
\end{tabular}

Table 1. Jet exhaust flow conditions in the JSI noise database.

\begin{tabular}{|c|c|c|c|c|c|c|c|}
\hline$h_{T E}($ inches $)$ & & & $x_{T E}($ inches $)$ & & & & \\
& 1.3 & 2.7 & 4 & 8 & 12 & 16 & 20 \\
\hline 0.0 & $3,5,7$ & $3,5,7$ & $A L L$ & $A L L$ & $A L L$ & $A L L$ & $A L L$ \\
\hline 0.1 & $3,5,7$ & $3,5,7$ & $3,5,7$ & $3,5,7$ & $3,5,7$ & $3,5,7$ & $3,5,7$ \\
\hline 0.2 & $3,5,7$ & $3,5,7$ & $3,5,7$ & $3,5,7$ & $3,5,7$ & $3,5,7$ & $3,5,7$ \\
\hline 0.3 & $3,5,7$ & $3,5,7$ & $3,5,7$ & $3,5,7$ & $3,5,7$ & $3,5,7$ & $3,5,7$ \\
\hline 0.5 & $3,5,7$ & $3,5,7$ & $A L L$ & $A L L$ & $A L L$ & $A L L$ & $A L L$ \\
\hline 0.7 & $3,5,7$ & $3,5,7$ & $3,5,7$ & $3,5,7$ & $3,5,7$ & $3,5,7$ & $3,5,7$ \\
\hline 1.0 & $3,5,7$ & $3,5,7$ & $A L L$ & $A L L$ & $A L L$ & $A L L$ & $A L L$ \\
\hline 1.4 & $3,5,7$ & $3,5,7$ & $3,5,7$ & $3,5,7$ & $3,5,7$ & $3,5,7$ & $3,5,7$ \\
\hline 1.5 & & & $A L L$ & $A L L$ & $A L L$ & $A L L$ & $A L L$ \\
\hline 1.9 & $3,5,7$ & $3,5,7$ & $3,5,7$ & $3,5,7$ & $3,5,7$ & $3,5,7$ & $3,5,7$ \\
\hline 2.0 & & & $A L L$ & $A L L$ & $A L L$ & $A L L$ & $A L L$ \\
\hline 2.5 & $3,5,7$ & $3,5,7$ & $A L L$ & $A L L$ & $A L L$ & $A L L$ & $A L L$ \\
\hline 3.0 & & & $A L L$ & $A L L$ & $A L L$ & $A L L$ & $A L L$ \\
\hline 3.2 & $3,5,7$ & $3,5,7$ & $3,5,7$ & $3,5,7$ & $3,5,7$ & $3,5,7$ & $3,5,7$ \\
\hline 4.0 & $3,5,7$ & $3,5,7$ & $A L L$ & $A L L$ & $A L L$ & $A L L$ & $A L L$ \\
\hline 5.0 & $3,5,7$ & $3,5,7$ & $A L L$ & $A L L$ & $A L L$ & $A L L$ & $A L L$ \\
\hline
\end{tabular}

Table 2. Surface lengths $\left(x_{T E}\right)$ and radial standoff distances $\left(h_{T E}\right)$ used to generate the empirical model. The numbers in each box represent the setpoint tested at that surface location ( $A L L$ indicates that all setpoints in Table 1). 


\section{Empirical Model for Engine Exhaust Noise}

The complexity of an empirical model is directly related to the number and range of variables included in that model. This complexity can be mitigated through the use of several component models that combine to make a prediction for the overall system. The complete exhaust noise spectra $\left(S P L_{T}\right)$ represents such a complex system containing multiple sources and effects. Therefore, the empirical engine exhaust noise model provides a framework to separate the different noise sources, each with one or more modifying effects, in an effort to avoid some of the problems inherent in a monolithic model. As currently constructed, the model contains two sources: jet mixing noise $\left(P_{m}\right)$ and JSI noise $\left(P_{d}\right)$. Each source has several modifying effects (e.g nozzle aspect ratio, flight effect, noise shielding) that can be added as data becomes available. Mathematically, the system might be written as:

$$
\begin{aligned}
S P L_{T}= & P_{m}\left(M_{a}, T_{T, R}\right)+G_{S / R}\left(x_{T E}, h_{T E}, M_{a}, T_{T, R}\right)+A\left(R, M_{a}, T_{T, R}\right) \oplus \\
& P_{d}\left(x_{T E}, h_{T E}, M_{a}, T_{R}\right)+B\left(x_{T E}, h, R, M_{a}, T_{T, R}\right)
\end{aligned}
$$

where $G_{S / R}$ accounts for the jet mixing noise shielding or reflected by the surface, $A$ accounts for the nozzle aspect ratio on the jet mixing noise $\left(P_{m}\right)$, and $B$ accounts for the nozzle aspect ratio on the jet-surface interaction noise $\left(P_{d}\right)$. These effects $\left(G_{S / R}, A, B\right)$ add directly to the source as decibels (logarithmic basis) while the sources are summed on an acoustic power basis (anti-logarithmically indicated by $\oplus$ ). Models for some of the terms in Equation 1 already exist: a model for the jet mixing noise source $\left(P_{m}\right)$ was developed by Khavaran and Bridges ${ }^{2,3}$ and a model for the effect of nozzle aspect ratio on the jet mixing noise $(A)$ has been developed by Bridges. ${ }^{9}$ The JSI15 model represents the jet-surface interaction source $\left(P_{d}\right)$.

Dividing the spectra into noise sources and effects is a convenient way structure an empirical model but it also imposes the requirement that those sources and effects can be separated in the underlying experimental data. In this case, the jet mixing noise can be measured independently. The aspect ratio effect on this isolated jet mixing noise can be separated by subtracting the results of two independent measurements, one using a round nozzle and the other a rectangular nozzle. The JSI noise source is more difficult because it can not be measured without some jet mixing noise and the noise shielding (or reflecting) effect of the surface. A methodology for separating the JSI noise source, relying in the coherent nature of a dipole point source, was used to develop the JSI14 model. ${ }^{8}$ That method is again used here to extract the coherent JSI noise from the total measured noise at each surface position, jet exit condition, and observer location where data were acquired.

\section{II.A. Jet-Surface Interaction Noise Source Model (JSI15)}

The JSI15 model is based on the idea that a characteristic spectra can be found for each surface position and jet exit condition. Theory, ${ }^{10-12}$ supported by experiments, ${ }^{6,13,14}$ have shown that JSI noise is dipolar in nature and, therefore, follows some basic amplitude and directivity scaling rules. The characteristic spectra equation provides a structure to the model that allows it to use these scaling rules while overcoming many of the signal-to-noise ratio problems inherent to the coherence based source separation method. ${ }^{8}$ The JSI15 model assumes a parabolic characteristic spectra in $d B / S t_{D j}$ for an observer at $\theta=90^{\circ}$, at a distance of $100 D_{j}$ from the nozzle exit (corrected to a lossless condition). Three coefficients are thus required to define the characteristic spectra as:

$$
P_{d}=C_{1}+C_{2} \log _{10}\left(f / F_{\text {peak }}\right)^{2}
$$

where $C_{1}$ gives the peak amplitude for the spectra, $C_{2}$ provides the spectra width, and $F_{\text {peak }}$ locates the frequency where the spectral peak occurs. These three coefficients are at the core of the JSI15 model.

The coefficients $C_{1}, C_{2}$ and $F_{\text {peak }}$ were initially determined by fitting Equation 2 to the JSI spectra extracted from the experimental data at each surface position and jet condition using a weighted least-squares fit procedure. The weighting function $(W(f))$ was determined by subtracting the jet mixing noise from the JSI noise at each frequency $\left(W(f)=P_{d}(f)-P_{m}(f)\right)$ so that the fit favor the region around the JSI noise peak amplitude. Considered together, the coefficients are functions of surface position and jet condition so that $C_{1} \rightarrow C_{1}\left(x_{T E}, h_{T E}, M_{a}, T_{T, R}\right), C_{2} \rightarrow C_{2}\left(x_{T E}, h_{T E}, M_{a}, T_{T, R}\right)$, and $F_{p e a k} \rightarrow F_{p e a k}\left(x_{T E}, h_{T E}, M_{a}, T_{T, R}\right) . \mathrm{A}$ model is then developed for each coefficient function. 


\section{II.A.1. Coefficient Models}

The three coefficient models are at the core of the JSI15 model and the key to these models is choosing a suitable nondimensionalization for each input parameter. First, the jet Mach number and temperature ratio as defined in experiments (Table 1) are nondimensional and, therefore, can be used directly. The surface length and standoff distance, however, have dimensions attached and must be nondimensionalized. An analysis of PIV data has shown that centerline jet velocity and turbulence profiles collapse across a range of jet Mach numbers and temperature ratios when the axial distance is nondimensionalized by the jet potential core length $\left(X_{c}\right){ }^{15}$ Thus, nondimensionalizing the surface length by jet potential core length has two effects on the modeling. First, the distance jet exit to the surface trailing edge is now relative to a point in development of the jet plume, accounting for jet Mach number and temperature ratio, rather than a fixed value. For example, a surface with $x_{T E} / D_{e}=7$ near an unheated $M_{a}=0.9$ jet $\left(X_{c} / D_{e} \approx 7\right)$ is equivalent to a surface with $x_{T E} / D_{e}=5$ near a $M_{a}=0.5$ jet at $T_{T, R}=2.75\left(X_{c} / D_{e} \approx 5\right)$; the surface is very close to the end of the potential core in both cases so it is reasonable to expect that the flow physics are also similar even though the jet conditions and physical dimensions are quite different. The second effect of this nondimensionalization is to introduce an adjustment for specific conditions that might exist in a given jet. The underlying database provides a good example here; a 12 inch pipe section was added upstream of the nozzle exit so that the surface could pass the jet rig upstream and sit directly at the nozzle lipline. An analysis of the flow data after the test showed that this additional section shortened the jet potential core length by approximately 1 jet diameter at $M_{a}=0.9 .{ }^{7}$ By accounting for this in the initial development, the model can be used with actual (or predicted) values and the surface will be at the same point relative to the jet flow as measured in the initial experiment.

If the surface length is best normalized by the jet potential core length, the surface standoff distance might be best nondimensionalized by some measure of the jet spread. Again, PIV data shows that the jet spread rate changes as the jet temperature ratio increases. ${ }^{15}$ However, unlike the potential core length the spread rate is more difficult to determine using relatively simple equations. Therefore, the standoff distance is nondimensionalized by the surface length $\left(h_{T E} / x_{T E}\right)$ to represent the angle between the nozzle lip and surface trailing edge. This nondimensionalization treats that the jet spread rate is a secondary effect (compared to the potential core length) and allows the fit to optimize the coefficients to account for changes to the JSI noise based on this fixed angle rather than a specific flow variable.

Once the independent variables are nondimensionalized a singular value decomposition (SVD) fit was used to define the coefficient models. All available data where $h_{T E} / x_{T E} \leq 1$ was used in the fit (the JSI noise is below the jet mixing noise when $h_{T E} / x_{T E}>1$ ). The basis functions for this fit were selected to capture the effect of changes within the known variable space and exhibit predictable behavior near the edges of the variable space. Thus, only second order polynomials and first order cross-terms were used. The lone exception is Mach number which is directly present only in an exponential term as JSI noise theory predicts that the amplitude scales with $U_{j}^{6}$ (note that $M_{a}$ and $T_{T, R}$ are also included in the surface length terms though the in the potential core length). Thus, the list of basis functions is:

$$
\begin{aligned}
F_{1} & =k \\
F_{2} & =x_{T E} / X_{c} \\
F_{3} & =\left(x_{T E} / X_{c}\right)^{2} \\
F_{4} & =h_{T E} / x_{T E} \\
F_{5} & =\left(h_{T E} / x_{T E}\right)^{2} \\
F_{6} & =\left(x_{T E} / X_{c}\right) *\left(h_{T E} / x_{T E}\right) \\
F_{7} & =T_{T, R} \\
F_{8} & =\left(T_{T, R}\right)^{2} \\
F_{9} & =\left(x_{T E} / X_{c}\right) * T_{T, R} \\
F_{10} & =\left(h_{T E} / x_{T E}\right) * T_{T, R} \\
F_{11} & =\log _{10}\left(M_{a}\right)
\end{aligned}
$$

The coefficients for each basis function are shown in Table 3. Note that observer angle (polar or azimuthal) 
are not included in the basis functions; the characteristic spectra is modeled at at $\theta=90^{\circ}, \phi=0^{\circ}$, where the peak JSI noise is observed, and simple dipole dipole directivity scaling $\left.\left(\cos ^{2}(\theta) \sin ^{2}(\phi)\right)^{16}\right)$ is applied to scale to other observer angles. Also note that the model finds that the amplitude scales as approximately $U_{j}^{6}$ spectral power, close to the theoretically expected value $\left(U_{j}^{5}\right.$ is found in $P a^{2} / S t_{D j}$ but there is an factor of $U_{j}$ in $S t_{D j}$ making $U_{j}^{6}$ in spectral power).

There are several steps in the modeling process where uncertainty or fit quality might be analyzed: the source separation, the least-squares fit of the characteristic spectra, or the SVD fit to the coefficients $C_{1}$, $C_{2}$, or $F_{\text {peak }}$. First, the quality of the coefficient models are evaluated by computing the root mean square deviation $(R M S D)$ between the modeled coefficient values and the input values (determined from the initial characteristic equation fit). The normalized root mean square deviation is also computed (NRMSD) to show how the model performs relative to the variable range.

The peak amplitude coefficient has two roles in the characteristic spectra equation: accurately predict the peak amplitude when the JSI noise is prevalent and capture the decreasing trend when the JSI noise is below the jet mixing noise. The peak amplitude model has a fit quality $R M S D=2.45$ across a range that includes JSI noise and jet mixing noise dominate configurations $\left(h_{T E} / x_{T E} \leq 1\right)$. Normalized by the range of amplitudes present in the database $\left(65 \leq C_{1} \leq 105\right)$, the $N R M S D=6.2 \%$. However, it should be noted here that the $C_{1}$ model returns a very large value when $h_{T E} / x_{T E}>1.5$ (the $\left(h_{T E} / x_{T E}\right)^{2}$ term dominates) so the JSI15 model should not be used in this region (where the JSI noise is very low in any event).

The coefficient model for the peak frequency $\left(F_{\text {peak }}\right)$ provides a fit quality of $R M S D=0.05$. However, because the range of values in the input data is small $\left(0.025 \leq F_{\text {peak }} \leq 0.31\right)$, the $N R M S D=17.5 \%$. There are two factors that influence the fit quality: configurations where the JSI noise is low relative to the jet mixing noise and configurations where there is a resonance between the flow and the surface. First, when the signal-to-noise ratio is low the characteristic spectra fitting routine finds it difficult to locate a defined peak. These points are then given a lower weight in the coefficient model resulting in a greater discrepancy in the overall model evaluation. However, as long as the $C_{1}$ coefficient model is correctly trending these points down in amplitude, the resulting characteristic spectra will be well below the jet mixing noise and the peak frequency will not matter. The second factor is the surface/flow resonance which has been previously documented. ${ }^{8,17,18}$ The resonance appears at specific combinations of flow and surface position but are not systematic or regular enough to captured by the second-order equations used by the coefficient models. However, they have the effect of shifting the peak frequency determined by the characteristic equation fit to slightly higher values thereby increasing the $R M S D$ metrics. Therefore, the fit quality metrics for the $F_{\text {peak }}$ coefficient model seem reasonable but direct comparisons between measured and modeled spectra are needed to confirm this.

The spectral width coefficient is the most dependent of the three coefficients on the spectral tails where the signal-to-noise ratio is relatively low. As a result the $C_{2}$ coefficient model has the most variation. This is reflected in the fit quality metrics where the $N R M S D=24.4 \%(R M S D=7.0 \%)$. It appears that much of this variation is due to cases where the peak JSI peak is not well defined, being in close proximity to the jet mixing noise. However, unlike the peak values the spectral width requires a fair number of points to determine and, in these cases, the low signal to noise ratio makes this difficult. Fortunately, this same problem means that the spectral width parameter will be slightly less important in the complete model as much of the spectral the tail will be below the jet mixing noise.

\section{II.A.2. Comparison Between Model and Data}

A second measure of model quality is the performance relative to the experimental data. Figure 2 shows the measured and modeled JSI and overall spectra at several standoff distances. The overall predicted spectra were made by combining the JSI15 model with an existing jet mixing noise model ${ }^{2,3}$ (the predicted jet mixing noise is shown for reference in figure 2). Notice first that the extracted JSI noise has a much flatter spectral peak than the peak modeled by the parabolic characteristic equation when $h_{T E} / x_{T E} \leq 0.125$. However, by fitting the peak data and the slope at the tails the JSI15 model provides the low frequency spectral lift observed in the data. Next, there is a peak between $0.7 \leq S t_{D j} \leq 2$, first at $h_{T E} / x_{T E}=0.05$ (figure figures 2(b)) and stronger at $h_{T E} / x_{T E}=0.125$ (figure 2(c)), that is not captured by the JSI15 model. These higher frequency peaks are caused a resonance phenomenon that occurs at specific combinations of flow condition and surface position; ${ }^{17,18}$ as transient features, a simple one peak model covering the entire range of surface positions and flow conditions will not capture these resonances. Finally, both the predicted and extracted JSI noise drops well below the jet mixing noise as the surface moves away from the jet out of 
the hydrodynamic region surrounding the plume. However, the extracted JSI noise approaches a minimum level around $h_{T E} / x_{T E}=0.25$ (note how similar the JSI spectra are at $h_{T E} / x_{T E}=0.625$ and 1.0). These surface positions are far enough from the jet that the coherence measured with the surface approaches the natural level of the isolated jet. The model, extrapolating using the trend line established at the closer surface positions, continues to decrease the amplitude as the surface moves farther away. This does not cause a problem in the overall noise prediction, however, as the jet mixing noise dominates at these surface positions, but it does appear in model quality metrics.

To quantify the performance of the JSI15 model, data within $10 \mathrm{~dB}$ of the peak amplitude were extracted from the measured and predicted spectra for all jet conditions and all surface positions where $h_{T E} / x_{T E} \leq 1$ (the $10 \mathrm{~dB}$ threshold minimized the surface shielding/reflecting effect which is not included in the model). The RMSD between the measured and modeled data was then calculated on the JSI noise alone (JSI15 model and extracted data) and the overall noise spectra; the results at four observer angles are shown in table 4. The RMSD for the JSI noise alone is heavily influenced by the peak shape of the extracted data and the low signal to noise ratio when the surface is away from the jet. This is particularly true at the downstream angle $\left(\theta=150^{\circ}\right)$ where the jet mixing noise is near its peak amplitude but the JSI noise is well below its peak (which is at $\theta=90^{\circ}$ ). When the jet mixing noise is included in the prediction, the relationship between the JSI and jet mixing noise sources is included and the overall spectra is predicted with a peak amplitude $2 \leq R M S D \leq 3.2$ and on OASPL within $1.1 \leq R M S D \leq 2.6$. Thus, caution should be used when predicting only the JSI noise because the uncertainty in (1) extracting the JSI spectra from the measured data and (2) the assumed parabolic spectral shape. However the combination of the JSI15 model with a jet mixing noise model gives an uncertainty level suitable for many system level studies.

The JSI15 model also supports hot jet conditions. The jet total temperature ratio enters the JSI15 model through dedicated basis functions and as part of the potential core length which is used to nondimensionalize the surface length. Figure 3 shows predicted overall spectra against measured data with a surface at $x_{T E}=$ 12 ", $h_{T E}=0$ " for two jet Mach numbers, $M_{a}=0.5$ and $M_{a}=0.9$, each at several jet total temperature ratios. First, note that there is some surface shielding effect in both cases above $S t_{D j} \leq 1$ illustrated by the slope change in the modeled spectra. The effect of jet temperature is relatively small at $M_{a}=0.5$ (Figure $3(\mathrm{a})$ ); there is a small lift at frequencies below the peak and a small reduction at frequencies above the peak as the jet temperature increases. The end of the jet potential core in these $M_{a}=0.5$ jets is upstream of the surface trailing edge (albeit only slightly in the unheated jet) and the close proximity minimizes the jet spreading effect that accompanies increasing temperature. At $M_{a}=0.9$ (figure 3(b)), the jet potential core is downstream of the surface trailing edge in the unheated case and near or upstream of the trailing edge in the hot jets. Similar to the behavior observed in isolated jets, ${ }^{19}$ there is a jump moving from the unheated jet condition to the the lowest heated jet condition and then changes with additional heat are then small. The effect of heat on the JSI noise is to narrow the spectral peak while shifting it to a lower frequency resulting in a reduction at higher frequencies compared to the unheated jet case. The JSI15 model captures both the initial change with heat and then agrees with the data that changes only slightly with additional jet temperature.

\begin{tabular}{|c|c|c|c|c|}
\hline Function & Term & $C_{1}$ & $C_{2}$ & $F_{\text {peak }}$ \\
\hline$F_{1}$ & $k$ & 91.20 & -25.91 & 0.355 \\
\hline$F_{2}$ & $x_{T E} / X_{c}$ & 19.15 & -8.45 & -0.176 \\
\hline$F_{3}$ & $\left(x_{T E} / X_{c}\right)^{2}$ & -5.05 & 4.70 & 0.024 \\
\hline$F_{4}$ & $h_{T E} / x_{T E}$ & -31.11 & 31.83 & -0.721 \\
\hline$F_{5}$ & $\left(h_{T E} / x_{T E}\right)^{2}$ & 15.47 & 0.41 & 0.321 \\
\hline$F_{6}$ & $\left(x_{T E} / X_{c}\right) *\left(h_{T E} / x_{T E}\right)$ & -10.65 & -8.54 & 0.205 \\
\hline$F_{7}$ & $T_{T, R}$ & -3.28 & -0.10 & -0.024 \\
\hline$F_{8}$ & $\left(T_{T, R}\right)^{2}$ & 0.51 & -0.21 & 0.000 \\
\hline$F_{9}$ & $\left(x_{T E} / X_{c}\right) * T_{T, R}$ & 0.29 & 0.37 & 0.006 \\
\hline$F_{10}$ & $\left(h_{T E} / x_{T E}\right) * T_{T, R}$ & 5.99 & -3.13 & 0.095 \\
\hline$F_{11}$ & $\log _{10}\left(M_{a}\right)$ & 49.21 & 0.00 & 0.000 \\
\hline
\end{tabular}

Table 3. Coefficients values for the 11 basis functions resulting from the SVD fit to data for $C_{1}, C_{2}$, and $F_{\text {peak }}$. 


\begin{tabular}{|c|c|c|c|c|c|}
\hline Polar Observer Angle $(\theta)$ & Source $(\mathrm{s})$ & $60^{\circ}$ & $90^{\circ}$ & $120^{\circ}$ & $150^{\circ}$ \\
\hline$R M S D$, Peak Amplitude & JSI Only & 6.3 & 5.7 & 4.6 & 14.8 \\
\hline$R M S D$, OASPL & JSI Only & 6.1 & 5.4 & 4.7 & 14.2 \\
\hline$R M S D$, Peak Amplitude & JSI + Jet Mixing & 2.5 & 2.1 & 3.2 & 2.0 \\
\hline$R M S D$, OASPL & JSI + Jet Mixing & 2.6 & 1.1 & 1.4 & 1.6 \\
\hline
\end{tabular}

Table 4. RMSD between the measured and predicted data. OASPL computed using data within 10 $\mathrm{dB}$ of the peak amplitude.

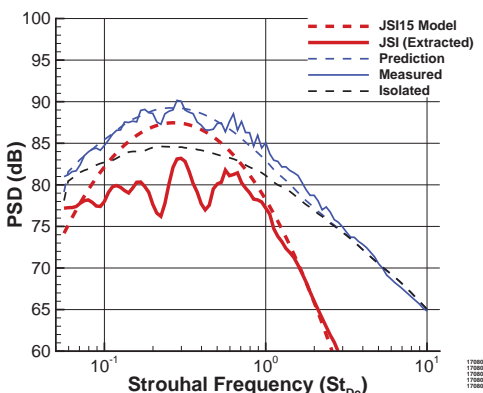

(a) $h_{T E} / x_{T E}=0.0$

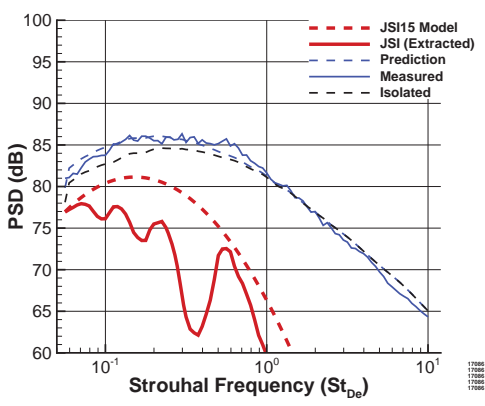

(d) $h_{T E} / x_{T E}=0.25$

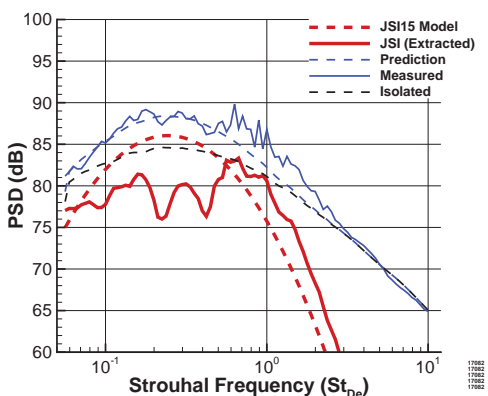

(b) $h_{T E} / x_{T E}=0.05$

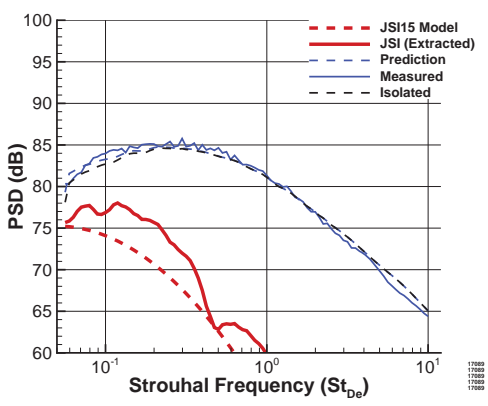

(e) $h_{T E} / x_{T E}=0.625$

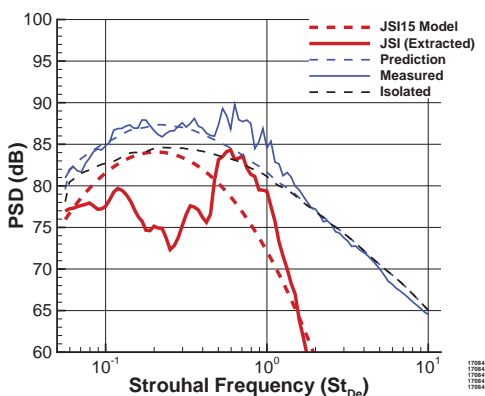

(c) $h_{T E} / x_{T E}=0.125$

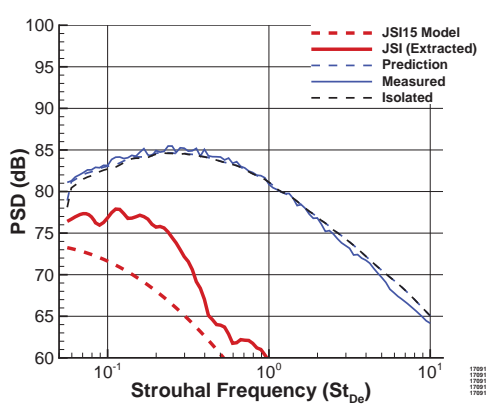

(f) $h_{T E} / x_{T E}=1.0$

Figure 2. Modeled and measured JSI and overall spectra at $\theta=90^{\circ}$ for the $M_{a}=0.5$, unheated jet condition with a surface at $x_{T E}=12 "\left(x_{T E} / X_{c}=0.38\right)$. The predicted isolated jet noise is also shown for reference.

\section{II.B. Effect of Nozzle Aspect Ratio on JSI Noise}

There a several factors that must be considered when predicting the noise produced by a jet exhaust; a partial list might include jet velocity, temperature, flight speed, nozzle shape, and the effect of nearby by surfaces. One template proposed for capturing some of these factors in an empirical model, represented in equation 1, separates the overall noise into primary sources and effects on those sources. Independent models can be used for these sources and effects with the combined results predicting the overall noise. Thus far the modeling efforts have focused on models associated with round jets (or the effect of multiple round jets ${ }^{20}$ ). The effect of nozzle aspect ratio is now considered.

The underlying paradigm considers the nozzle aspect ratio an effect that modifies the accompanying source by adding (or subtracting) directly on a logarithmic (dB) basis. This effect is then a difference that might determined by subtracted two measured spectra. The aspect ratio correction for the isolated jet $(A$ in equation 1) can be determined in exactly this way; far-field noise data is acquired at the same jet condition using a round nozzle and various rectangular nozzles which can then be subtracted and the result fit to a mathematical function to from the effect model. Figure 4 shows how this process works for an isolated rectangular jet; the effect is extracted from the rectangular nozzle spectra from the round nozzle spectra (figure $4(\mathrm{a})$ ), the effect is modeled by a fit at each observer angle and $1 / 3$-octave frequency band, and the 


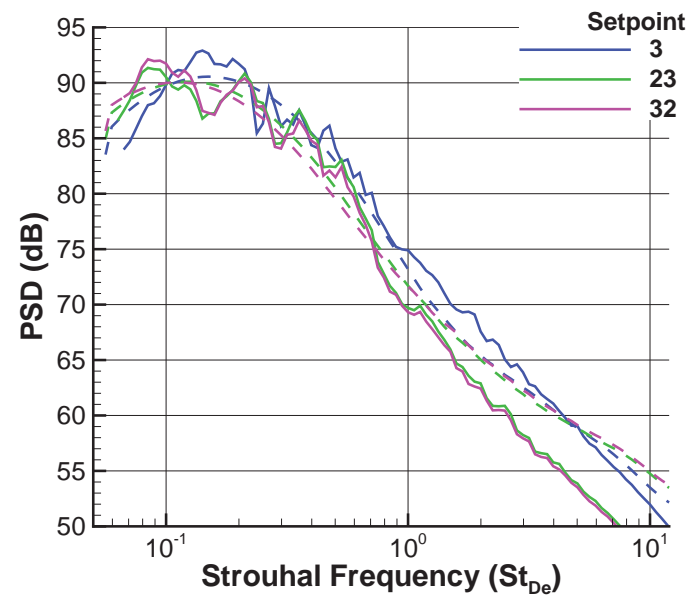

(a) $M_{a}=0.5$

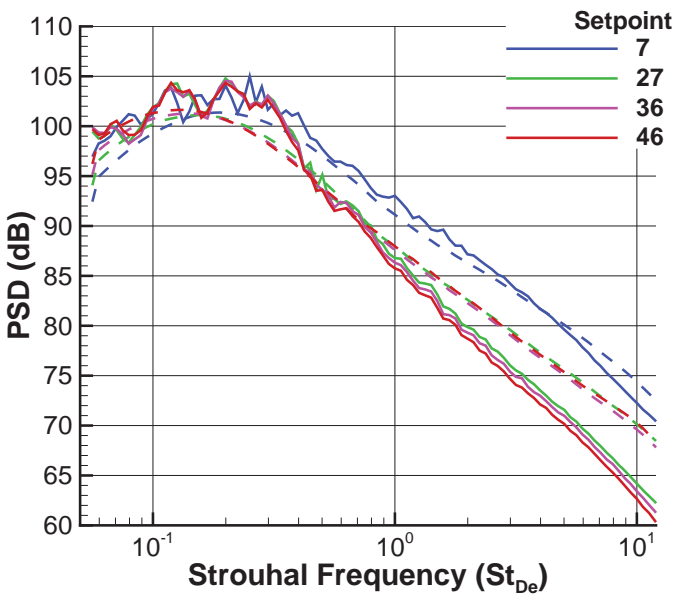

(b) $M_{a}=0.9$

Figure 3. Modeled (dashed) and measured (solid) overall noise predictions at $\theta=90^{\circ}$ for $x_{T E}=12$ ", $h_{T E}=0$ " at $M_{a}=0.5$ (left) and $M_{a}=0.9$ (right) and several jet total temperature ratios.

effect model is combined with the existing source model to produce the modeled spectra (figure 4(a)). As the data shows and the model reflects, the effect of a rectangular nozzle is to move energy some energy from low frequencies to higher frequencies as the aspect ratio increases. Thus, this is an effective way to model something that changes a source when the change can be separated from the source itself.

The effect of a rectangular nozzle on the jet-surface interaction noise is more complex. It can not be independently measured independently of the jet mixing noise, also a function of nozzle aspect ratio, and therefore must be separated by other means. The coherence of the surface trailing edge dipole was used to extract the JSI noise for the round jet, a process that was used again with the rectangular nozzle data. However, the coherence extracted data was the source in the round nozzle case but here the difference between the coherence extracted round nozzle and rectangular nozzle data is needed to model the effect. A reasonable measure of the effect can be extracted for the longer surface close to the jet where the JSI source is strong in both round and rectangular spectra. When the signal to noise ratio is higher or when there is a significant frequency shift, the subtracting the JSI spectra leads to wide swings between frequencies which are very difficult to model. This is complicated further by the occasional resonance behavior which appear stronger in the higher aspect ratio rectangular nozzle data. It is, therefore, necessary to find another method for creating a first order model for a rectangular jet near a surface.

The JSI15 model presented for round jets nondimensionalized the surface length by the jet potential core length. This works in part because there are several models for the potential core length of a single stream round jet (e.g. [15],[21]) and the coefficients can be tuned to work with any one of them. These convenient relationships are not common for rectangular jets. However, recent data published by Bridges and Wernet ${ }^{22}$ measure the potential core length for the 2:1, 4:1, and 8:1 nozzles using the Jet-Surface Interaction Tests operating at setpoint 7 allowing investigation of how the JSI15 source model performs directly for rectangular jets. First, figure 5(a) show the result of simply running the JSI15 model with the potential core lengths of the rectangular nozzles. By only adjusting for the potential core length, the amplitude is fairly close but the spectral peak occurs a at frequency that is too low. The jet mixing noise produced by high aspect ratio nozzles can scale with slot height $(H)$ rather then equivalent diameter. Therefore, the JSI noise peak frequency was shifted by a factor of $H / D_{e}$ and the model was rerun again using the potential core length of the rectangular jet. These results (figure 5(b)) show improvement by this frequency shift particularly in the 8:1 nozzle data. The 8:1 aspect ratio nozzle has a potential core length of $X_{c} \approx 3 D_{e}$ so the $x_{T E}=2.7 "$ surface trailing edge is not quite halfway to its end. Figure 6 shows who the spectra changes if the surface trailing edge is downstream of the potential core of the 8:1, near the end for the 4:1, and still upstream of the $2: 1$. In this case, the frequency shift is clearly too much for the $8: 1$ and $4: 1$ nozzles but not the 2:1. Finally, a $x_{T E}=12$ " surface (the longest in this dataset, figure 7) is near the end of the potential core of the 2:1 nozzle and even farther downstream for the 4:1 and 8:1. At this point, the frequency shift is not necessary 
for any of the nozzles and, in fact, the spectra is not too different from the round nozzle case. It appears, then, that the JSI15 model developed using data from a round nozzle can be applied to rectangular nozzles if the potential core length of the rectangular jet is given and a frequency shift is applied if the surface length is less than (or near) the end of the potential core. At some point downstream the JSI noise produced by the rectangular jet is very similar to the equivalent round jet and the frequency adjustment is not required. Overall the effect of nozzle aspect ratio on JSI noise appears relatively small and this simple scaling therefore represents a first order approximation to the effect.

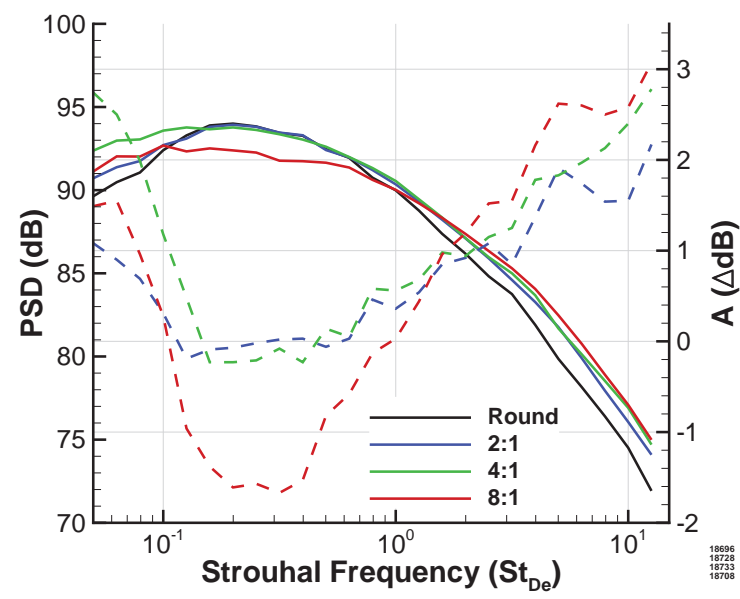

(a) Effect Extraction

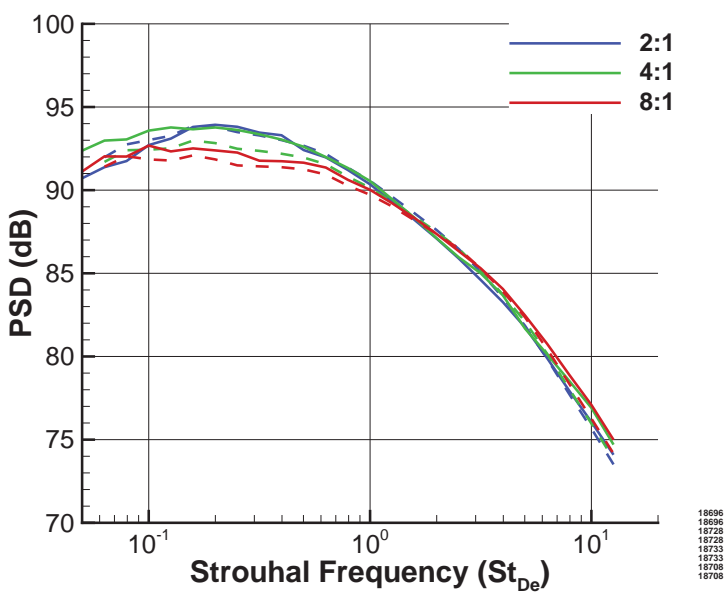

(b) Modeled

Figure 4. Separation of the nozzle aspect ratio effect ( $A$ in equation 1 ) in an isolated $M_{a}=0.9$ unheated jet (left) and the reconstruction of the spectra using the jet mixing noise model for a round jet and a separate model for the aspect ratio effect.

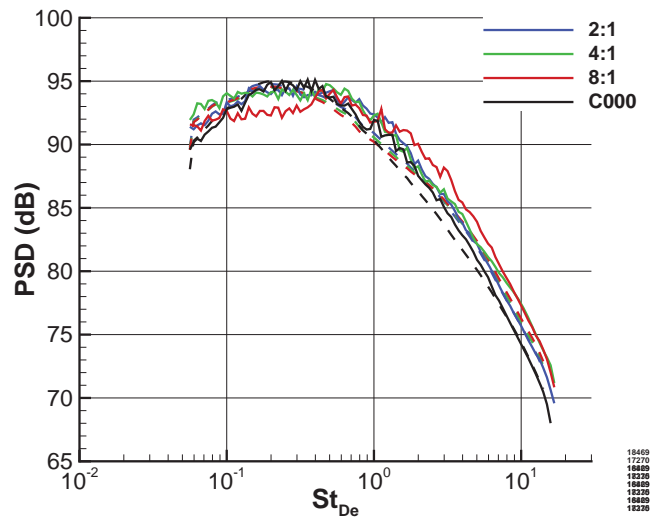

(a) $X_{c}$ Adjusted



(b) $X_{c}$ and $F_{\text {peak }}$ Adjusted

Figure 5. Spectra modeled using the JSI15 model for unheated $M_{a}=0.9$ rectangular jets with a surface at $x_{T E}=2.7 "$ and $h=0.2 "$.

\section{Conclusions}

An empirical model for the jet-surface interaction (JSI) noise source has been developed. The model covers unheated and hot jet conditions $\left(1 \leq T_{T, R} \leq 2.7\right)$ in the subsonic range $\left(0.5 \leq M_{a} \leq 0.9\right)$, surface lengths $0.6 \leq x_{T E} / D_{j} \leq 10$, and surface standoff distances $\left(0 \leq h_{T E} / x_{T E} \leq 1\right)$. The JSI noise model uses only second-order polynomials to give predictable behavior between data point and near the edges of the 


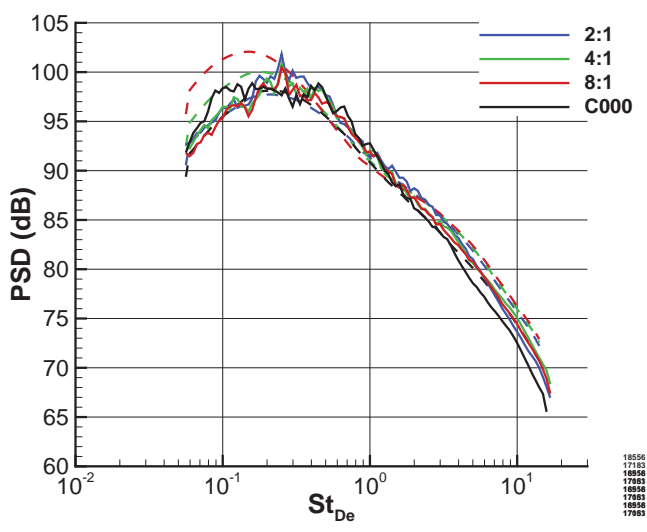

(a) $X_{c}$ Adjusted

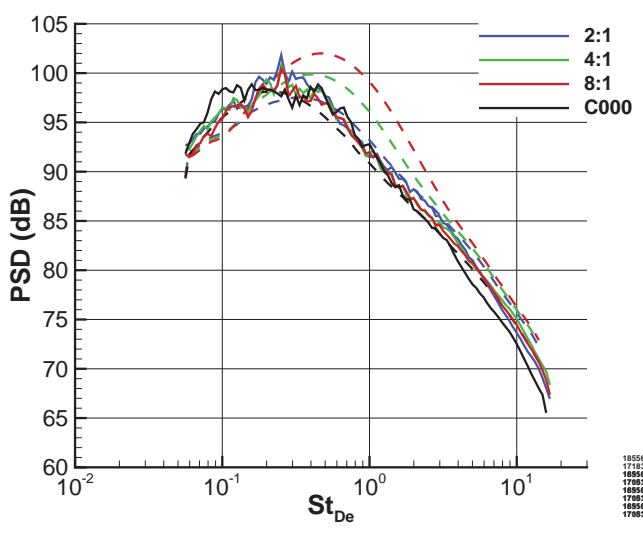

(b) $X_{c}$ and $F_{\text {peak }}$ Adjusted

Figure 6. Spectra modeled using the JSI15 model for unheated $M_{a}=0.9$ rectangular jets with a surface at $x_{T E}=8 "$ and $h=0.2 "$.

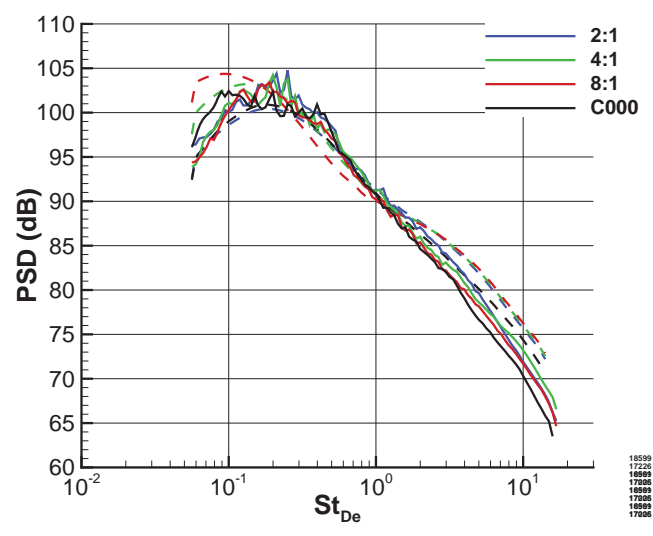

(a) $X_{c}$ Adjusted

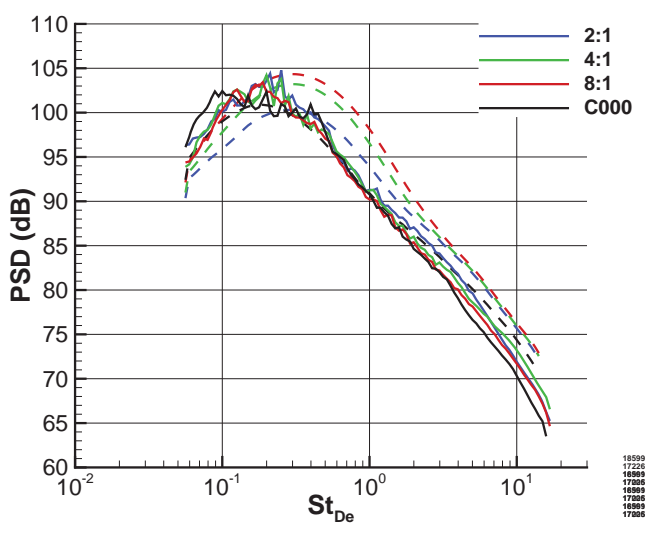

(b) $X_{c}$ and $F_{\text {peak }}$ Adjusted

Figure 7. Spectra modeled using the JSI15 model for unheated $M_{a}=0.9$ rectangular jets with a surface at $x_{T E}=12$ " and $h=0.2 "$. 
variable space. The overall model uses 31 coefficients determined by a singular value decomposition fit to the experimental data. Analysis of the fit quality and comparison with the source data indicates that the JSI noise source model is suitable for many system level studies.

A first-order correction to the JSI source model that accounts for the effect of nozzle aspect ratio has been explored. The JSI noise associated with a rectangular jet appears to have a peak amplitude similar to the equivalent round jet if adjusted for differences in potential core length. A shift in the peak frequency, scaling by slot height rather than equivalent exit diameter, was also applied when the surface was longer than the potential core length. These simple corrections appear to account for effects in nozzle up to an 8:1 aspect ratio and may, by themselves, be used in some applications. However, these ideas still need to be refined into a usable model and an uncertainty analysis done to quantify the model performance.

Jet-surface interaction noise is a potential problem in many proposed concept aircraft. Trends toward integrating the engine more closely to the airframe to increase operating efficiency or minimize sonic boom indicate that JSI noise will remain an issue that must be considered in the initial system level optimization studies.

\section{Acknowledgements}

This work was supported by the NASA Fundamental Aeronautics Program, Fixed Wing and High Speed Projects. The author would like to thank James Bridges for modeling the isolated rectangular jet effect and for reviewing this work. The author would also like thank Rick Bozak for reviewing this work.

\section{References}

\footnotetext{
${ }^{1}$ Khavaran, A., Bridges, J., and Georgiadis, N., "Prediction of Turbulence-Generated Noise in Unheated Jets, Part 1: JeNo Technical Manual (Version 1.0)", NASA/TM 2005-213827, 2005.

${ }^{2}$ Khavaran, A. and Bridges, J., "Development of jet noise power spectral laws using SHJAR data", AIAA 2009-3378, 2009.

${ }^{3}$ Khavaran, A. and Bridges, J., "SHJAR jet noise data and power spectral laws", NASA/TM 2009-215608, 2009.

4, Brown, C., "Jet-Surface Interaction Test: Far-Field Noise Results", ASME GT2012-69639, 2012.

${ }^{5}$ Podboy, G., "Jet-Surface Interaction Test: Phased Array Noise Source Localization Results", ASME GT2012-69801, 2012.

${ }^{6}$ Brown, C. , "Jet-Surface Interaction Test: Far-Field Noise Results", J. Eng. Gas Turbines Power, 135(7), Jun. 2013.

${ }^{7}$ Brown, C., "Jet-Surface Interaction Test: Flow Measurement Results", AIAA 2014-3198, 2014.

${ }^{8}$ Brown, C., "Developing an Empirical Model for Jet-Surface Interaction Noise", AIAA 2014-0878, 2014.

${ }^{9}$, Bridges, J., "Acoustic Measurements of Rectangular Nozzles with Bevel, AIAA 2012-2252, 2012.

${ }^{10}$ Curle, N., "The influence of solid boundaries upon aerodynamic sound", Proc. Roy. Soc. of London, 231A, pp. 505-514, 1955.

${ }^{11}$ Ffowcs-Williams, J.E. and Hall, L.H., "Aerodynamic sound generation by turbulent flow in the vicinity of a scattering half plane", J. Fluid Mech., 40(4), pp. 657-670, 1970.

${ }^{12}$ Crighton, D.G. and Leppington, F.G., "Scattering of aerodynamic noise by a semi-infinite compliant plate", J. Fluid Mech., 43(4), pp. 721-736, 1970.

${ }^{13}$ Head, R.W. and Fisher, M.J., "Jet/Surface Interaction Noise: Analysis Of Farfield Low Frequency Augmentation of Jet Noise Due To The Presence Of A Solid Surface", AIAA 1976-502, 1976.

${ }^{14}$ Lawrence, J.L.T., Azarpeyvand, M., and Self, R.H., "Interaction between a Flat Plate and a Circular Jet

${ }^{15}$ Bridges, J. and Wernet, M. P., "The NASA Subsonic Jet Particle Image Velocimetry (PIV) Dataset", NASA/TM-2011216807, 2011.

${ }^{16}$ Russell, D. A., Titlow, J. P., and Bemmen, Y.-J., "Acoustic monopoles, dipoles, and quadrapoles: An experiment revisited", Am. J. Phys., 67(8), Aug. 1999.

${ }^{17}$ Zaman, K.B.M.Q., Fagan, A., Clem, M., Brown, C., "Resonant Interaction of a Rectangular Jet with a Flat-plate", AIAA 2014-0877, 2014.

${ }^{18}$ Bridges, J., "Noise from Aft Deck Exhaust Nozzles - Differences in Experimental Embodiments", AIAA 2014-0876, 2014.

${ }^{19}$ Brown, C. and Bridges, J., "Small Hot Jet Acoustics Rig Validation", NASA/TM 2006-214234, 2006.

${ }^{20}$ Bozak, R., "Twin Jet Effects: Experiment and Model", AIAA 2014-2890, 2014.

${ }^{21}$ Witze, P., "Centerline velocity decay of compressible free jets", AIAA J. 12(4), pp 417-418, 1974.

${ }^{22}$ Bridges, J. and Wernet, M.P., "Turbulence Measurements of Rectangular Nozzles with Bevel", to be presented at the 2015 AIAA SciTech Conference, 5-9 January, 2015, Kissimmee, FL.
} 